\title{
Effect of Varying Repositioning Frequency on Prevention of Pressure Injuries in Nursing Home Residents: TEAM-UP Cluster-Randomized Clinical Trial Results
}

Tracey L. Yap ( $\sim$ tracey.yap@duke.edu )

Duke University

Susan D. Horn

University of Utah School of Medicine

Phoebe D. Sharkey

Loyola University Maryland

Tianyu Zheng

University of Utah

Nancy Bergstrom

The University of Texas Health Science Center at Houston

Cathleen Colon-Emeric

Duke University

Valerie K. Sabol

Duke University

Jenny Alderden

University of Utah

Winston Y Yap

Carroll County Memorial Hospital

Susan M. Kennerly

East Carolina University

\section{Research Article}

Keywords: Compliance, Cueing, Geriatrics, Nursing, Nursing Home, Pressure Injury, Pressure Ulcer, Prevention, Repositioning

Posted Date: August 2nd, 2021

DOI: https://doi.org/10.21203/rs.3.rs-717425/v1

License: (c) (1) This work is licensed under a Creative Commons Attribution 4.0 International License. Read Full License

Version of Record: A version of this preprint was published at Advances in Skin \& Wound Care on January 19th, 2022. See the published version at https://doi.org/10.1097/01.ASW.0000817840.68588.04. 


\section{Abstract}

Background: Pressure injuries (Prls) cause pain and infection, lengthen institutional care, raise healthcare costs, and hasten death in nursing home $(\mathrm{NH})$ residents. Repositioning residents to offload pressure on tissues is the most common prevention strategy, yetthe optimal interval remains uncertain.

Objective: Investigate clinical effectiveness of three nursing-home-wide repositioning intervals (2-,3-, or 4-hours) without compromising Prl incidence in four weeks in adult nursing home residents.

Design, Setting, and Participants: Anembedded pragmatic cluster randomized controlled trial was conducted in $9 \mathrm{NHs}$ from a large proprietary system located in 34 states. Each $\mathrm{NH}$ was randomly assigned to one of three $\mathrm{NH}$-wide repositioning interval Arms (every 2, 3, or 4 hours). Data were provided for 12-month Baseline and 4-week Intervention during the Turn Everyone And Move for Ulcer Prevention (TEAM-UP) study. Intervention residents were without current Prls, had Prlrisk (Braden Score) $\geq 10$ (low, mild, moderate, or high risk; not severe risk), and used viable 7" high-density foam mattresses.

Methods: Each Arm included three NHs with assigned single repositioning interval as standard care during the 4-week Intervention. Mandatory staff education sessions were provided.A wireless patient monitoring system, using wearable singleuse patient sensors, cued staff by displaying resident repositioning need on conveniently placed monitors.Primary outcome was Prl incidence; secondary outcome was staff repositioning compliance fidelity.

Results: Eleven hundred residents from 9 NHs were fitted with sensors; 108 of these were ineligible for some analyses due to missing Baseline data. Effective sample size included 992 residents (mean age 78 years [SD13]; 63\% female). Pressure injury incidence during the Intervention was $0.0 \%$ compared to $5.24 \%$ in Baseline, even though Intervention resident clinical risk scores were significantlyhigher $(\mathrm{p}<0.001)$. Repositioning compliance for 4-hour repositioning interval $(95 \%)$ was significantly better than $2-(80 \%)$ or $3-(90 \%)$ hours $(p<.001)$.

Conclusions: This study contributes to understanding alternativerepositioning intervalssuggestingcurrent 2-hour protocolscan be relaxed for many without compromising Prl development. Causal link was not established between three repositioning interval treatments and primary outcome-Prls; however, no new Prls developed. Cueing staff may benefit repositioningimplementation; compliance improved as repositioning interval lengthened.

Trial Registration: (ClinicalTrials.gov Identifier: NCT02996331).Date: 16/05/2017

\section{Background}

Pressure injuries (localized damage to skin and/or underlying tissues over bony prominences from pressure or shear forces) are common, yet seemingly intractable geriatric conditions that are mostly preventable complications in nursing home residents, ${ }^{1}$ who are typically older, with multiple comorbidities, mobility challenges, and have severely compromised health. $2,3,4$ Annual pressure injury prevalence in long-stay (7.3\%) and short-stay (2.3\%) residents ${ }^{5}$ and incidence rates up to $59 \%$ are reported, ${ }^{1}$ hence, the emphasis on pressure injury prevention. Pressure injuries have severe negative impacts on patients (pain, infection, and death), health care settings, and insurers, ${ }^{1,6}$ potentially exceeding 26 billion dollars annually. ${ }^{7}$

Assessment of pressure injury risk is standard practice in nursing homes, ${ }^{8-10}$ but prevention has proved elusive. ${ }^{11}$ There is limited evidence for pressure injury prevention, ${ }^{12}$ with support surfaces and manual repositioning having been the focus. Highspecification foam alternatives to standard hospital foam mattresses were found to significantly reduce pressure injury incidence. $2,13,14$ Repositioning ("turning people to change body position"11) reduces pressure duration and tissue hypoxia, has theoretical appeal as a preventive approach, but also increases nursing workload and disrupts sleep. ${ }^{1,11}$ No conclusive evidence for either optimal repositioning frequency ${ }^{13,14}$ or angle/position has emerged, ${ }^{11}$ relegating practice settings to use status quo 2-hour intervals based on 60 year-old findings. ${ }^{15}$ Staff compliance with 2-hour repositioning is challenging to achieve; impact of cueing staff to reposition on-time is not established. 
The repositioning intervention of the clinical trial protocol for the Turn Everyone And Move for Ulcer Prevention (TEAM-UP) trial ${ }^{16}$ was derived from the International Pressure Injury Prevention Guidelines ${ }^{1}$ every 2 -hour standard for repositioning residents that allows individualization of repositioning intervals up to 4 hours; the protocol was used by all participating nursing homes. The TEAM-UP trial examines 2-, 3-, and 4-hour repositioning intervals prompted by wearable patient position sensors on 28-day pressure injury incidence among nursing home residents using viable 7" high-density foam mattresses and having pressure injury risk scores (Braden Score $\geq 10$ (low, mild, moderate, or high risk; not severe risk)). Final study results provide evidence of overall repositioning effectiveness among three repositioning intervals.

\section{Methods}

This study aimed to determine whether repositioning interval can be extended from 2 to 3 or 4 hours for $\mathrm{NH}$ residents without compromising pressure injury incidence. An embedded pragmatic cluster randomized trial design was selected to represent the real-world nursing home environment that includes: 1) a diverse representative study population, 2) an intervention that could be incorporated easily into routine clinical workflow as standard of care, 3) outcomes (pressure injury and compliance) important to decision-makers, 4) comprehensive data collected through standard documentation in an electronic health record within the health care setting, and 5) design input from health care stakeholders. ${ }^{17}$ Prior to nursing home selection, 3 Arms (Arm 1 = 2-hour, Arm 2 = 3-hour, Arm 3 = 4-hour) were determined with planned assignment of $3 \mathrm{NHs}$ to each Arm applying a randomized sequencing of the Arm assignments according to the chronological order identified for NHs. The repositioning intervals were implemented in chronological sequence after completing one round of Arm 1, Arm 2, and Arm 3 to ensure all 3 intervals could be safely implemented; then, a predetermined sequence was followed resulting in Nursing Homes 1, 6, 8 in Arm 1; Nursing Homes 2, 4, 9 in Arm 2; and Nursing Homes 3, 5, 7 in Arm 3. Nine nursing homes from a large proprietary system in 34 states met the eligibility requirements, accepted the invitation to participate, and were assigned to one of the three nursinghome-wide repositioning interval Arms as described above. The magnitude of within-cluster dependence was quantified by the Intraclass Correlation Coefficient and the precision of this measure was quantified by its confidence interval.

A patient monitoring system cued staff (during the Intervention) to reposition residents and tracked events. Four-week incidence of new pressure injury was compared among the three Arms, controlling for resident characteristics and staffing levels. Details of the trial design were published previously. ${ }^{16}$

\section{Study Setting, Residents, and Procedures}

All Medicare-certified nursing homes providing intermediate and skilled nursing care within the proprietary company $(n=473)$ were eligible for randomization. Inclusion criteria for nursing homes were: >100 beds; standard use of high density foam mattresses determined to be viable or replaced within two months of study implementation; adequate internet bandwidth capacity to support real-time data collection and storage; and full electronic health record capability including activities of daily living, laboratory, and radiology results. The requirement for nursing homes to have full electronic record capabilities was essential to facilitate data collection and ensured that the study would have robust data sets. Eighty-three nursing homes meeting eligibility requirements were invited to participate in the trial, which required mandatory staff in-service training to explain the study and patient monitoring system implementation. Sample size power requirement (95\%) was satisfied by the first 9 nursing homes assenting to participate based on total residents to be recruited. The 9 study nursing homes 1) had the same standard care delivery policies, 2) were of typical size and characteristics of the other eligible sites, 3) signed implementation agreements, 4) received a nominal stipend to support project implementation, and 5) were randomized via a predetermined random sequencing procedure ${ }^{16}$ to one of three repositioning intervals (Arms).

Eligible study participants met the following criteria: $\geq 18$ years, without pressure injuries (on admission or within 72 hours), Braden score $\geq 10$ (assessed weekly), and without adhesive allergy or other clinical contraindications (paranoia, dermatitis, personal defibrillator garment and monitor, or 'do-not-turn' order). Residents at severe pressure injury risk (Braden $<10)$ were excluded because of unique repositioning and surface needs. Available electronic historical data for study residents were retrieved for the 12-month Baseline period prior to the Intervention start at each nursing home. Residents with both Intervention

Page $3 / 21$ 
and Baseline electronic health record, Minimum Data Set, and nursing home Risk Management System data formed the effective sample for pre-post analyses.

\section{INTERVENTION}

Each Arm included three nursing homes assigned a nursing-home-wide repositioning interval (2, 3, or 4 hours) during the 4week Intervention. A wireless patient monitoring system using a wearable resident specific sensor worn on upper chest, tracked position/movement and cued staff compliance with prescribed nursing-home-wide repositioning interval. ${ }^{18} \mathrm{Health}$ Insurance Portability and Accountability Act compliant visual cues displayed each resident's time-stamped repositioning history and current status on unit desk and hallway screens. Patient monitoring system fidelity checks (6/week) ensured accuracy.

Pressure injury prevention care other than repositioning intervals was provided in all three Arms in accordance with International Pressure Injury Prevention Guidelines ${ }^{1}$ (head-of-bed elevation, position angle, and duration and use of pillows/wedges to maintain position, turning sheets, and lift devices as appropriate). ${ }^{1}$ Staff assisted non-bedfast residents to stand/move/reposition and used preventive seating cushions as needed. All residents/families received information about the study, repositioning protocol, and their right to refuse care and/or receive a more frequent repositioning interval.

\section{Outcomes}

The primary outcome was pressure injury incidence during the Intervention. Daily and weekly nursing home staff skin assessments were recorded using nursing home system policies. ${ }^{1}$ Certified Nursing Assistants observed skin daily over bony prominences, between skin folds, in genitalia/buttocks areas, and at sensor sites. Change in skin appearance was reported to licensed staff with oversight for repositioning, safety, weekly skin care checks, and electronic health record documentation related to pressure injury status (stage and manifestations). Safety algorithms were published previously. ${ }^{16}$

The secondary outcome was fidelity of staff repositioning compliance tracked by wearable patient sensors, enhanced by nursing home mandated in-service training for full- and part-time registered nurses, licensed practical nurses, and Certified Nursing Assistants (79\% participation rate). The required education focused on pressure injury etiology, Braden scale risk assessment refresher, evidence-based prevention practices, repositioning benefits, roles and responsibilities, staff workflow, trial protocol, and patient monitoring system information. A researcher visited the nursing home each shift during Week 1 and at least daily during Weeks 2-4 to ensure the patient monitoring system was functioning, answer staff questions, and stock supplies.

\section{Assessments}

Nursing homes and eligible residents were assessed retrospectively for Baseline period (maximum 365 days) before Intervention start date and prospectively for 4-week Intervention period. The nursing home parent company provided all electronic health record, Minimum Data Set, and Risk Management System data for the full 28-day Intervention period regardless of number of days repositioning was monitored. Assessments of all eligible residents were extracted from electronic health record, Minimum Data Set, or nursing home Risk Management System. The electronic health record provided demographic characteristics, medical diagnoses as International Classification of Disease-9 (Baseline period data) and/or International Classification of Disease-10 codes (most frequently occurring codes were grouped into the most common diagnosis categories), height/weight, vital signs, and laboratory data. Electronic health record data were supplemented by the Minimum Data Set, a federally mandated, comprehensive, standardized assessment of nursing home residents' functional and health needs conducted quarterly and/or at condition change. Nursing home assessments (location, specialty units, Medicare-certified beds, census, occupancy, staff hours, and payor type) for Intervention and Baseline were extracted as reported to Centers for Medicare and Medicaid Services. Braden assessments produced the only data that for which the data extraction/collection schedule varied between Baseline and Intervention periods. During Baseline, Braden risk scores (10-12= high, 13-14 = moderate, 15-18 = mild, 19-23 = low) were assessed on admission, weekly x 4, quarterly, and upon condition change. During Intervention, Braden risk was assessed weekly; no residents were withdrawn because of Braden score $\leq 9$. 
Pressure injury incidence for eligible residents during the 12-month Baseline period was extracted retrospectively from the nursing home Risk Management System and supplemented by the Minimum Data Set and electronic health record, as previously described. ${ }^{16}$ Pressure injury incidence was determined through standard weekly licensed nursing staff skin assessments for the 4-week Intervention, directly reported to researchers, and documented as an adverse event through the nursing home company's Risk Management System. Researchers ensured study fidelity by randomly verifying assessments and receipt in real-time of secure email notification triggered by the reported event.

The patient monitoring system served as a repositioning fidelity measure, which assessed several factors for each resident: days with active sensors worn, number of Turn Alert cues, Turn Alert overdue hours, and degree angle of repositioning with $+/-2.5 \%$ accuracy. Turn Alert cues appeared on screens to notify staff that a resident had not had a position change and was due for repositioning within the allocated interval. Turn Alert overdue hours counted time beyond prescribed repositioning interval that a resident remained in the same position. The 24-hour on-time repositioning compliance was calculated as (1 - [\# Turn Alert overdue hours for period of interest]/[Total hours monitored for period of interest]) and indicated the degree to which the expected repositioning interval was being achieved.

\section{Statistical Analysis}

Primary analyses of pressure injury outcomes were performed according to intention-to-treat principle. Analyses were conducted using Statistical Analysis Software (SAS version 9.4).

\section{Analysis of Intervention Outcomes}

The initial analysis plan to test whether the pressure injury rate during Intervention was higher for 3-hour or 4-hour repositioning compared to 2 -hour is reported elsewhere. ${ }^{16}$ Overlap between the $95 \%$ confidence intervals of rates of pressure injury and the 2-hour repositioning would confirm the hypothesis for no group difference. However, given that no pressure injuries developed during the Intervention, the trial's original analysis plan was modified to report the trial's characteristics and pre-post comparative analyses by testing Baseline (pre-test, 2 hour repositioning) versus Intervention (post-test, 3 and 4 hour protocols) pressure injury rates.

\section{Additional Analyses}

Analysis of Differences in Baseline and Intervention Risk. Propensity score Logistic Regression analysis based on Baseline data was used to account for an imbalance in pressure injury risk associated with significant differences in nursing home and resident characteristics across Arms. Adequacy of the final model fit was ensured by generating a $70 \%$ random sample to train/build the model and a $30 \%$ random sample to validate the model. Regression coefficients from our fitted training dataset model provided unbiased risk predictions of developing a pressure injury during the Intervention. Contribution of each variable to likelihood of developing a pressure injury was determined by odds ratios generated from the model. C statistics were used to assess goodness of fit.

Differences in First Braden and Mean Braden total risk scores across Arms within the Intervention period were compared separately using either ANOVA or Chi Square analyses. Paired t-tests evaluated differences in Mean Braden total risk scores between Baseline and Intervention cohorts by Arm. Two-sided tests ( $p$-value $<0.05)$ were used for all analyses.

\section{Power Analyses}

Statistical power and sample size analyses are published elsewhere ${ }^{16}$. The expected pressure injury incidence rate for this study's 4-week Intervention was 3.5\% based on the TURN study's ${ }^{13}$ highest rate (for moderate-risk patients) during that 3-week intervention. Target sample size was 951 residents ( $\geq 317$ per Arm) to detect minimum detectable effect size of 0.38 difference between study arms with a power of 0.95 based on a one-sided rather than a two-sided test to determine if pressure injury incidence with 3 or 4 hour repositioning frequency was greater than with 2-hour repositioning; detection of a decrease in pressure injury was not of concern. Stopping boundaries were maintained during the trial as described in the Data Safety 
Monitoring Plan; no safety concerns were identified, and the trial was completed. Power was adjusted post-Intervention after taking into consideration the Intraclass Correlation Coefficient of the nursing homes in the three treatment Arms. Intraclass Correlation Coefficient and its confidence interval were calculated.

\section{Results}

Repositioning intervals were implemented in the randomly ordered sequence as planned to ascertain whether repositioning interval could be extended from 2 to 3 or 4 hours for $\mathrm{NH}$ residents. No Prls developed among participating residents even though the pre-Intervention Prl incidence at the $9 \mathrm{NHs}$ ranged from 2.3 to 18.3 percent. Intervention results are described related to nursing home and resident characteristics and primary and secondary outcomes. Additional analyses were performed to examine Prl risk and repositioning compliance.

\section{Nursing Homes}

Characteristics of the three nursing homes in each Arm during Baseline are presented in Table 1; the Intraclass Correlation Coefficient and its confidence interval are $0.056(\mathrm{Cl}=-0.78,0.89)$. Nursing homes were primarily suburban, with 126-238 Medicare-certified beds; some nursing homes had dementia and/or transitional resident specialty units. Average census ranged from approximately 143 to 162 residents, with Medicare-certified bed occupancy between 79\%-90\%. Certified Nursing Assistants provided most care hours to residents, who were primarily Medicaid supported. 
Characteristics of Nursing Homes $(\mathrm{N}=9)$ at Baseline, by Arm.

\begin{tabular}{|c|c|c|c|}
\hline \multirow[t]{2}{*}{ Nursing Home Characteristics* } & Arm 1 & Arm 2 & Arm 3 \\
\hline & $\begin{array}{l}2 \mathrm{hrt}(\mathrm{N}= \\
3)\end{array}$ & $\begin{array}{l}3 \mathrm{hrt}(\mathrm{N}= \\
3)\end{array}$ & $\begin{array}{l}4 \mathrm{hrt}(\mathrm{N}= \\
3)\end{array}$ \\
\hline Location $-\mathrm{U}=$ Urban, $\mathrm{S}=$ Suburban & $S, S, S$ & $S, U, S$ & $S, U, S$ \\
\hline Specialty units $-\mathrm{D}=$ Dementia Unit, $\mathrm{T}=$ Transitional Care Unit & $\begin{array}{l}\text { None, } \mathrm{D} / \mathrm{T} \\
\text { D }\end{array}$ & $\mathrm{T}, \mathrm{D}, \mathrm{T}$ & $\mathrm{D} / \mathrm{T}, \mathrm{T}, \mathrm{T}$ \\
\hline No. of Medicare certified beds per facility - mean (range) & $\begin{array}{l}181.0 \\
(180-183)\end{array}$ & $\begin{array}{l}178.7 \\
(176-180)\end{array}$ & $\begin{array}{l}180.3 \\
(126-238)\end{array}$ \\
\hline Patient Census $\ddagger-$ mean (SD) & $\begin{array}{l}143.2 \\
(37.9)\end{array}$ & $161.5(4.3)$ & $\begin{array}{l}159.1 \\
(47.7)\end{array}$ \\
\hline$\%$ Occupancy $\ddagger$ (census certified beds) - mean (SD) & $79.2(17.6)$ & $90.3(2.3)$ & $88.5(3.0)$ \\
\hline \multicolumn{4}{|c|}{$\begin{array}{l}\text { No. of staff hours per resident day } \ddagger \text { (no. of monthly hours/NH monthly } \\
\text { census, by staff category) - mean }\end{array}$} \\
\hline Registered Nurse & $1.6(0.20)$ & $2.1(0.53)$ & $2.4(0.53)$ \\
\hline Licensed Practical Nurse & $2.9(0.70)$ & $2.6(0.49)$ & $2.7(0.93)$ \\
\hline Certified Nursing Assistant & $6.5(1.40)$ & $7.5(0.36)$ & $7.6(0.57)$ \\
\hline \multicolumn{4}{|l|}{ Resident Payor Type Mean (\% Coverage by Payor Type) } \\
\hline Managed care & $3.8(2.6 \%)$ & $7.9(4.9 \%)$ & $11.2(7.0 \%)$ \\
\hline Medicaid & $\begin{array}{l}112.7 \\
(78.7 \%)\end{array}$ & $\begin{array}{l}131.9 \\
(81.7 \%)\end{array}$ & $\begin{array}{l}115.4 \\
(72.5 \%)\end{array}$ \\
\hline Medicare A & $\begin{array}{l}17.9 \\
(12.5 \%)\end{array}$ & $10.1(6.2 \%)$ & $\begin{array}{l}18.4 \\
(11.6 \%)\end{array}$ \\
\hline Private pay & $4.5(3.2 \%)$ & $5.4(3.4 \%)$ & $3.3(2.1 \%)$ \\
\hline Other payor & $4.3(3.0 \%)$ & $6.2(3.8 \%)$ & $10.8(6.8 \%)$ \\
\hline \multicolumn{4}{|l|}{ * Percentages may not sum to 100 because of rounding. } \\
\hline \multicolumn{4}{|l|}{ † Indicates repositioning interval for each intervention arm. } \\
\hline$\neq$ Baseline values are for the 6 -month period prior to the interv & & & \\
\hline
\end{tabular}

\section{Residents}

From May 2017 to October 2019, 1100 residents were fitted with sensors; 108 of these were ineligible for some analyses due to missing Baseline data or other disqualifying conditions. Effective sample size included 992 residents. Nursing home enrollment and randomization, resident assessment and allocation, follow-up, and analysis are shown in Fig. 1 developed according to the cluster trials extension of the CONSORT Statement.

Table 2 presents characteristics of 1100 residents allocated to the three Intervention Arms, 108 residents excluded from the pre-post analyses, and 992 residents analyzed by Arms. The 108 excluded residents did not form a pressure injury and were significantly younger $(p<0.001)$, primarily male $(p=0.002)$, had less cerebrovascular disease $(p=0.004)$, gastroesophageal reflux disease $(p=0.026)$, Alzheimer's Disease and Related Dementias $(p<0.001)$, and difficulty swallowing $(p<0.001)$, and more hypertension $(p<0.001)$ and diabetes $(p=0.003)$ than the 992 analyzed Intervention population. 
Table 2

Demographic and Clinical Characteristics of Residents Allocated to Intervention ( $N=1100)$ and Included in Pre-Post Analyses $(\mathrm{N}=992)$.

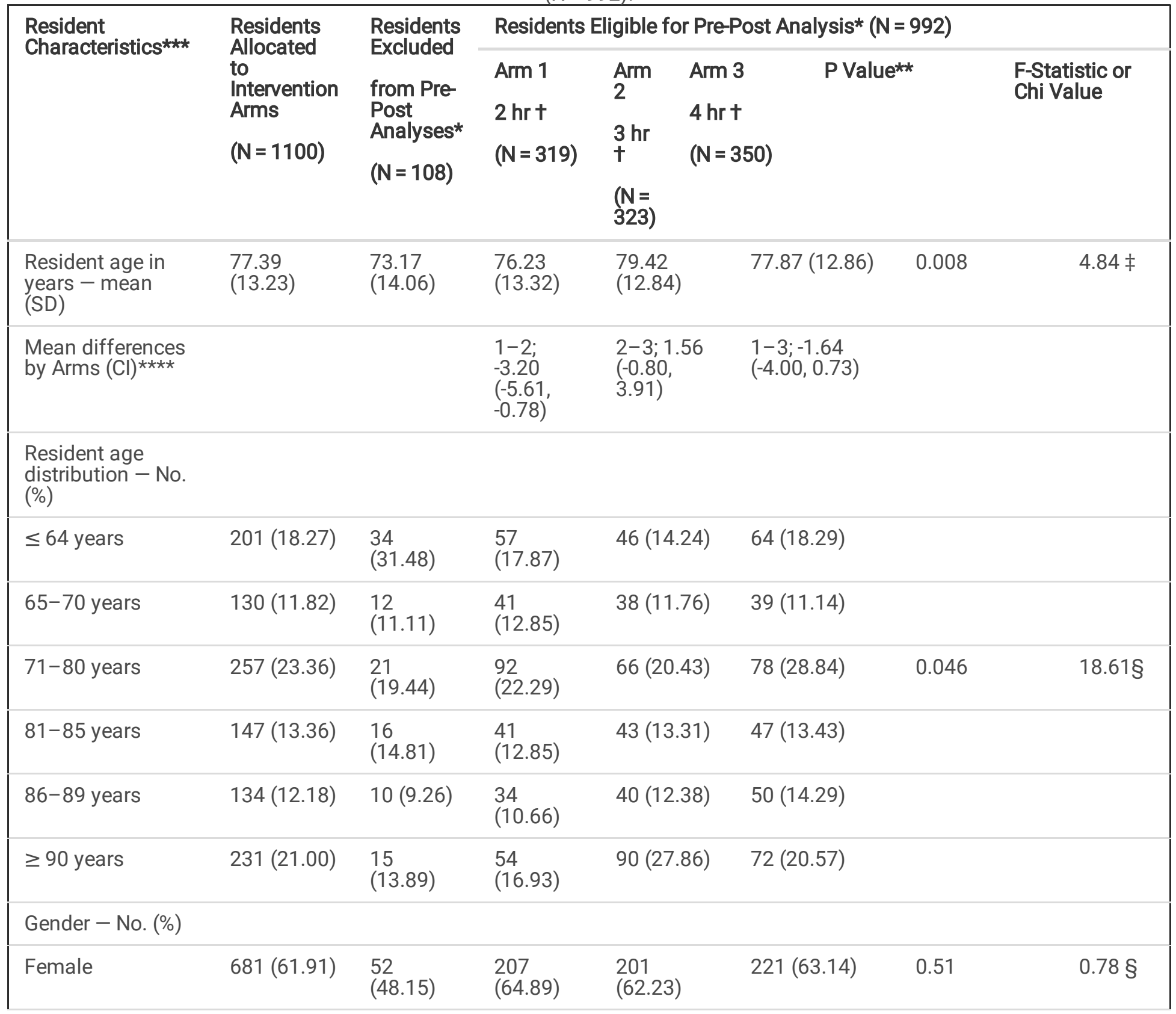

* Eligibility for Pre-Post analyses required data from both Baseline and Intervention time periods. The 108 residents not included in Pre-Post analyses did not have Baseline data.

** Denotes Arm Differences.

$\star \star \star$ Percentages may not sum to 100 because of rounding.

$\star \star \star \star$ Mean Difference displayed pairwise: Arm "x"- Arm "y"; Value of actual pairwise mean difference; Confidence Interval (a, b)

† Indicates repositioning interval for each Intervention Arm. Each arm included 3 nursing homes.

$\ddagger$ ANOVA used to test for differences among Arms.

$\S$ Chi-square used to test for differences among Arms.

$\iint \%$ American Indian/Alaska Native $=0 . \%$ More than one race $=0 . \%$ Native Hawaiian or Other Pacific Islander $=0$. 


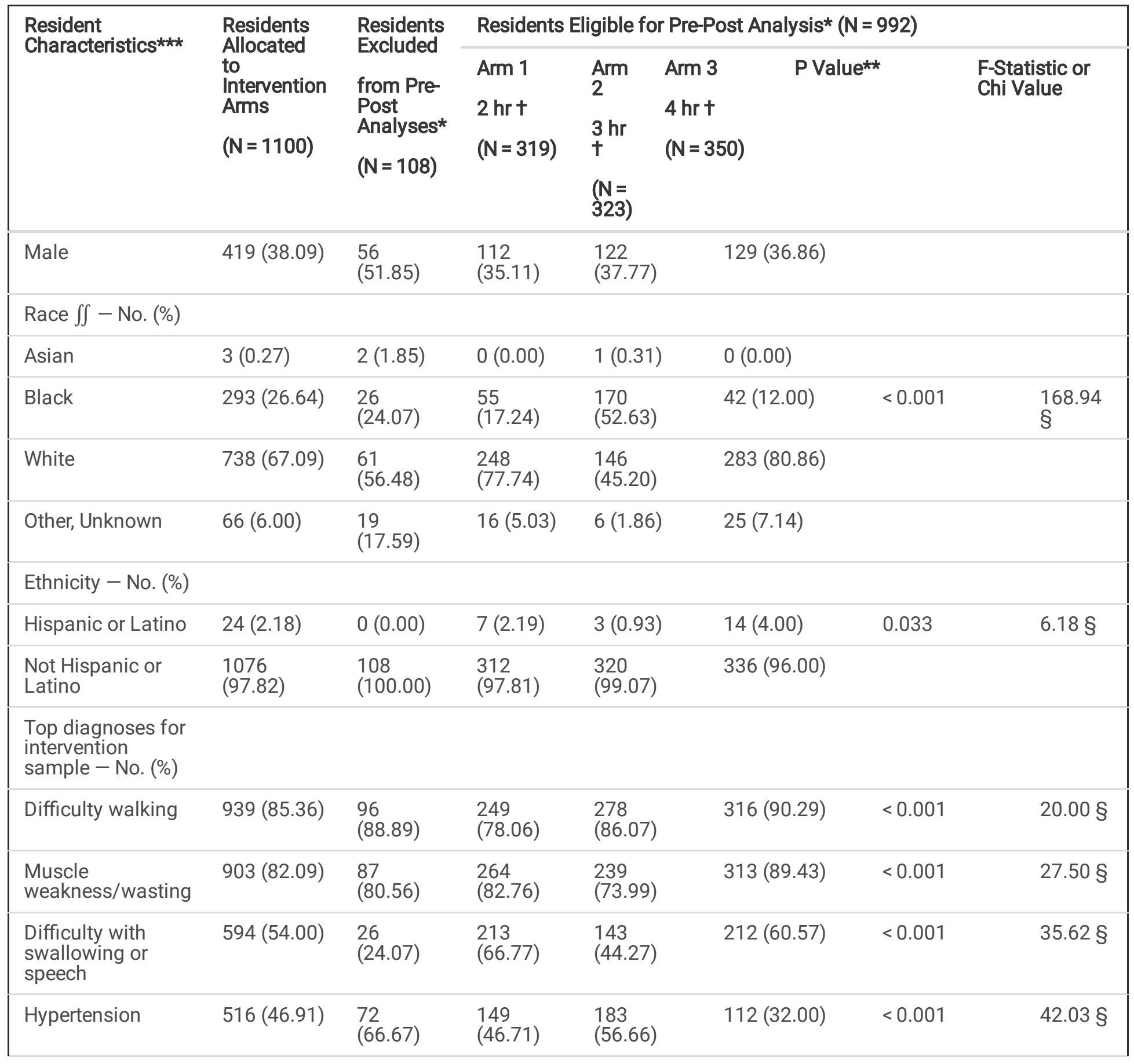

* Eligibility for Pre-Post analyses required data from both Baseline and Intervention time periods. The 108 residents not included in Pre-Post analyses did not have Baseline data.

** Denotes Arm Differences.

*** Percentages may not sum to 100 because of rounding.

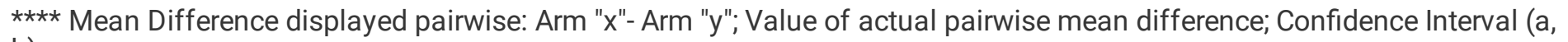
b)

† Indicates repositioning interval for each Intervention Arm. Each arm included 3 nursing homes.

$\ddagger$ ANOVA used to test for differences among Arms.

$\S$ Chi-square used to test for differences among Arms.

$\iint \%$ American Indian/Alaska Native $=0 . \%$ More than one race $=0 . \%$ Native Hawaiian or Other Pacific Islander $=0$. 


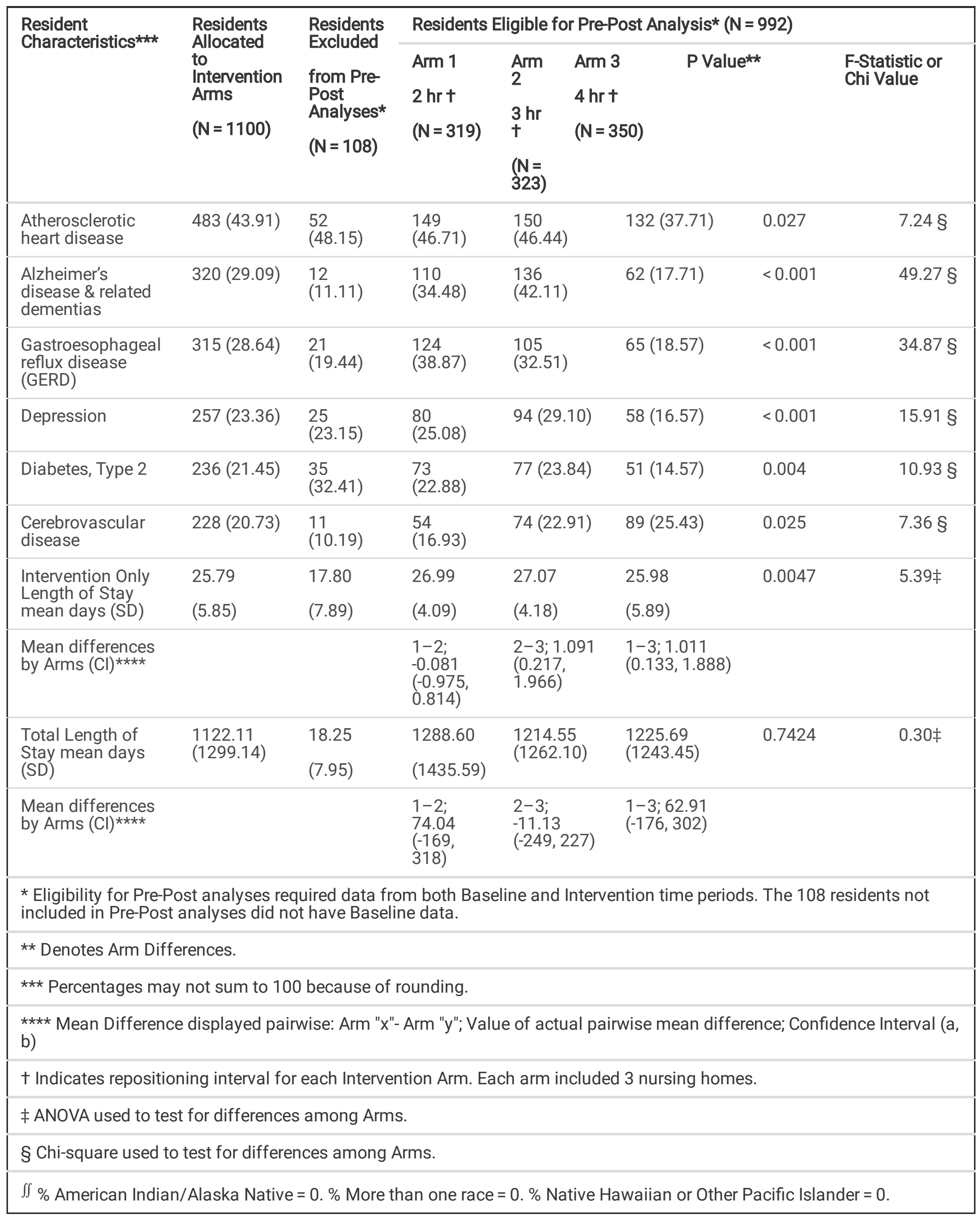

Differences in age, race, ethnicity, diagnosis categories, and Intervention length of stay $(n=992)$ were statistically significant across Arms (Table 2); this imbalance was addressed using propensity analyses (Table 3). Black residents in Arm 2 comprised 
$53 \%$ in comparison to $<18 \%$ in Arms 1 and 3 . Fifty-two residents ( $27 \%$ of whom were Black) had one or more incident Baseline pressure injuries that healed prior to Intervention start (untabled). Total Length of Stay reflects the time from admission that could have occurred during or before Baseline period until the Intervention end date or resident discharge from nursing home. Length of stay for only the Intervention time period (Intervention Only Length of Stay) was one day shorter in Arm 3, which was statistically significant, but a single day difference is not considered clinically relevant to the outcome. 
Table 3

Pre-Post Comparison of Predicted and Observed Risk of Pressure Injury for Residents at Baseline and Intervention by Arm ( $\mathrm{N}=$ 992).

\begin{tabular}{|c|c|c|c|c|c|c|c|c|}
\hline \multirow{2}{*}{$\begin{array}{l}\text { Resident } \\
\text { Characteristics* }\end{array}$} & \multicolumn{4}{|c|}{ Baseline Period † } & \multicolumn{4}{|c|}{ Intervention Period $¥$} \\
\hline & Arm 1 & Arm 2 & Arm 3 & P value/ & Arm 1 & Arm 2 & Arm 3 & P value/ \\
\hline & $\begin{array}{l}2 \mathrm{hr} \S \\
(\mathrm{N}= \\
319)\end{array}$ & $\begin{array}{l}3 \mathrm{hr} \S \\
(\mathrm{N}= \\
323)\end{array}$ & $\begin{array}{l}4 \mathrm{hr} \S \\
(\mathrm{N}= \\
350)\end{array}$ & $\begin{array}{l}\text { F-Statistic } \\
\text { or }\end{array}$ & $\begin{array}{l}2 \mathrm{hr} \S \\
(\mathrm{N}= \\
319)\end{array}$ & $\begin{array}{l}3 \mathrm{hr} \S(\mathrm{N} \\
=323)\end{array}$ & $\begin{array}{l}4 \mathrm{hr} \S \\
(\mathrm{N}= \\
350)\end{array}$ & $\begin{array}{l}\text { F-Statistic } \\
\text { or }\end{array}$ \\
\hline & & & & Chi Square & & & & Chi Square \\
\hline $\begin{array}{l}\text { First Braden Total } \\
\text { Score - mean (SD) }\end{array}$ & $\begin{array}{l}18.40 \\
(3.04)\end{array}$ & $\begin{array}{l}18.10 \\
(2.62)\end{array}$ & $\begin{array}{l}18.21 \\
(2.57)\end{array}$ & $\begin{array}{l}0.38 / 0.97 \\
\iint\end{array}$ & $\begin{array}{l}17.48 \\
(3.39)\end{array}$ & $\begin{array}{l}17.34 \\
(2.94)\end{array}$ & $\begin{array}{l}17.37 \\
(3.02)\end{array}$ & $0.84 / 0.18 \iint$ \\
\hline $\begin{array}{l}\text { Mean differences by } \\
\text { Arms (Cl) })^{\star \star \star}\end{array}$ & $\begin{array}{l}1-2 ; \\
0.299 \\
(-0.210 \\
0.808)\end{array}$ & $\begin{array}{l}2-3 \\
-0.115 \\
(-0.612 \\
0.382)\end{array}$ & $\begin{array}{l}1-3 \\
0.184 \\
(-0.315 \\
0.683)\end{array}$ & & $\begin{array}{l}1-2 ; \\
0.139 \\
(-0.439 \\
0.717)\end{array}$ & $\begin{array}{l}2-3 \\
-0.028 \\
(-0.593 \\
0.537)\end{array}$ & $\begin{array}{l}1-3 \\
0.111 \\
(-0.456 \\
0.678)\end{array}$ & \\
\hline $\begin{array}{l}\text { 19-23 (Low risk) - } \\
\text { No. (\%) }\end{array}$ & $\begin{array}{l}167 \\
(52.4)\end{array}$ & $\begin{array}{l}145 \\
(44.9)\end{array}$ & $\begin{array}{l}176 \\
(50.3)\end{array}$ & $0.09 / 11.07$ & $\begin{array}{l}138 \\
(43.3)\end{array}$ & $\begin{array}{l}109 \\
(33.8)\end{array}$ & $\begin{array}{l}134 \\
(38.3)\end{array}$ & $0.003 / 20.01$ \\
\hline $\begin{array}{l}15-18 \text { (Mild risk) - } \\
\text { No. (\%) }\end{array}$ & $\begin{array}{l}119 \\
(37.3)\end{array}$ & $\begin{array}{l}149 \\
(46.1)\end{array}$ & $\begin{array}{l}153 \\
(43.7)\end{array}$ & & $\begin{array}{l}110 \\
(34.5)\end{array}$ & $\begin{array}{l}158 \\
(48.9)\end{array}$ & $\begin{array}{l}150 \\
(42.9)\end{array}$ & \\
\hline $\begin{array}{l}\text { 13-14 Moderate risk) } \\
\text { - No. (\%) }\end{array}$ & $23(7.2)$ & $23(7.1)$ & $18(5.1)$ & & $\begin{array}{l}44 \\
(13.8)\end{array}$ & $43(13.3)$ & $\begin{array}{l}36 \\
(10.3)\end{array}$ & \\
\hline $\begin{array}{l}10-12 \text { (High risk) - } \\
\text { No. (\%) }\end{array}$ & $10(3.1)$ & $6(1.9)$ & $3(0.9)$ & & $\begin{array}{l}27 \\
(8.5)\end{array}$ & $13(4.0)$ & $\begin{array}{l}30 \\
(8.6)\end{array}$ & \\
\hline $\begin{array}{l}\text { Mean Braden Total } \\
\text { Score - mean (SD) }\end{array}$ & $\begin{array}{l}18.07 \\
(2.77)\end{array}$ & $\begin{array}{l}17.73( \\
2.25)\end{array}$ & $\begin{array}{l}17.89 \\
(2.51)\end{array}$ & $0.23 / 1.45 \iint$ & $\begin{array}{l}17.53 \\
(3.32)\end{array}$ & $\begin{array}{l}17.51 \\
(2.79)\end{array}$ & $\begin{array}{l}17.25 \\
(2.94)\end{array}$ & $0.40 / 0.92 \iint$ \\
\hline $\begin{array}{l}\text { Mean differences by } \\
\text { Arms }(\mathrm{Cl})^{\star \star \star}\end{array}$ & $\begin{array}{l}1-2 ; \\
0.338 \\
(-0.128 \\
0.804)\end{array}$ & $\begin{array}{l}2-3 ; \\
-0.157 \\
(-0.613 \\
0.298)\end{array}$ & $\begin{array}{l}1-3 \\
0.181 \\
(-0.276 \\
0.638)\end{array}$ & & $\begin{array}{l}1-2 ; \\
0.014 \\
(-0.545 \\
0.574)\end{array}$ & $\begin{array}{l}2-3 \\
0.265 \\
(-0.282 \\
0.812)\end{array}$ & $\begin{array}{l}1-3 ; \\
0.280 \\
(-0.269 \\
0.828)\end{array}$ & \\
\hline $\begin{array}{l}\text { 19-23 (Low risk) - } \\
\text { No. (\%) }\end{array}$ & $\begin{array}{l}135 \\
(42.3)\end{array}$ & $\begin{array}{l}104 \\
(32.2)\end{array}$ & $\begin{array}{l}132 \\
(37.7)\end{array}$ & $0.03 / 14.01$ & $\begin{array}{l}126 \\
(39.5)\end{array}$ & $111(34.4)$ & $\begin{array}{l}113 \\
(32.3)\end{array}$ & $0.001 / 24.08$ \\
\hline $\begin{array}{l}\text { 15-18 (Mild risk) - } \\
\text { No. (\%) }\end{array}$ & $\begin{array}{l}139 \\
(43.6)\end{array}$ & $\begin{array}{l}181 \\
(56.0)\end{array}$ & $\begin{array}{l}167 \\
(47.7)\end{array}$ & & $\begin{array}{l}118 \\
(37.0)\end{array}$ & $\begin{array}{l}160 \\
(49.5)\end{array}$ & $\begin{array}{l}163 \\
(46.6)\end{array}$ & \\
\hline $\begin{array}{l}\text { 13-14 Moderate risk) } \\
- \text { No. (\%) }\end{array}$ & $\begin{array}{l}39 \\
(12.2)\end{array}$ & $\begin{array}{l}37 \\
(11.5)\end{array}$ & $\begin{array}{l}44 \\
(12.6)\end{array}$ & & $\begin{array}{l}52 \\
(16.3)\end{array}$ & 43 (13.3) & $\begin{array}{l}40 \\
(11.4)\end{array}$ & \\
\hline $\begin{array}{l}\text { 10-12 (High risk) - } \\
\text { No. (\%) }\end{array}$ & $6(1.9)$ & $1(0.3)$ & $7(2.0)$ & & $\begin{array}{l}23 \\
(7.2)\end{array}$ & $9(2.8)$ & $\begin{array}{l}34 \\
(9.7)\end{array}$ & \\
\hline $\begin{array}{l}\text { Pressure Injury } \\
\text { Propensity Score for } \\
\text { Sample during Time } \\
\text { Period \|| }\end{array}$ & 0.044 & 0.066 & 0.048 & $\begin{array}{l}< \\
0.001 / 17.81\end{array}$ & 0.044 & 0.063 & 0.045 & $\begin{array}{l}< \\
0.001 / 13.94 \\
\iint\end{array}$ \\
\hline $\begin{array}{l}\text { Mean differences by } \\
\text { Arms }(\mathrm{Cl})^{\star \star \star}\end{array}$ & $\begin{array}{l}1-2 ; \\
-0.023 \\
(-0.032 \\
-0.013)\end{array}$ & $\begin{array}{l}2-3 \\
0.019 \\
(0.009 \\
0.028)\end{array}$ & $\begin{array}{l}1-3 \\
-0.004 \\
(-0.013 \\
0.005)\end{array}$ & & $\begin{array}{l}1-2 ; \\
-0.018 \\
(-0.028 \\
-0.009)\end{array}$ & $\begin{array}{l}2-3 \\
0.017 \\
(0.008 \\
0.026)\end{array}$ & $\begin{array}{l}1-3 \\
-0.001 \\
(-0.010 \\
0.008)\end{array}$ & \\
\hline $\begin{array}{l}\text { Pressure Injury } \\
\text { Incidence Rate - No. } \\
\text { (\%) during Time } \\
\text { Period, } n=992- \\
\text { mean of } 9 \text { sites }\end{array}$ & $52(5.24)$ & & & & $0(0)$ & & & \\
\hline
\end{tabular}




\begin{tabular}{|c|c|c|c|c|c|c|c|c|}
\hline \multirow{2}{*}{$\begin{array}{l}\begin{array}{l}\text { Resident } \\
\text { Characteristics* }\end{array} \\
\text { Pressure Injury } \\
\text { Incidence Rate - No. } \\
(\%) \text { during Time } \\
\text { Period n = 992 - } \\
\text { mean by Arm }\end{array}$} & \multicolumn{4}{|c|}{ Baseline Period t } & \multicolumn{4}{|c|}{ Intervention Period ¥ } \\
\hline & $\begin{array}{l}13 \\
(4.08)\end{array}$ & $\begin{array}{l}23 \\
(7.12)\end{array}$ & $\begin{array}{l}16 \\
(4.57)\end{array}$ & $0.18 / 1.74 \iint$ & $0(0)$ & $0(0)$ & $0(0)$ & NA ** \\
\hline $\begin{array}{l}\text { Mean differences by } \\
\text { Arms }(\mathrm{Cl})^{\star \star \star *}\end{array}$ & $\begin{array}{l}1-2 ; \\
-3.046 \\
(-7.174- \\
1.083)\end{array}$ & $\begin{array}{l}2-3 ; \\
2.549 \\
(-1.486- \\
6.585)\end{array}$ & $\begin{array}{l}1-3 \\
-0.496 \\
(-4.545- \\
3.552)\end{array}$ & & & & & \\
\hline \multicolumn{9}{|l|}{$\begin{array}{l}\text { Prl Incidence Rate by } \\
\text { Mean Braden Total } \\
\text { Score Categories }\end{array}$} \\
\hline $\begin{array}{l}\text { 19-23 (Low risk) - } \\
\text { No. (\%) }\end{array}$ & $3(2.2)$ & $3(2.9)$ & $1(0.8)$ & \multirow[t]{4}{*}{$0.47 / 5.629$} & $0(0)$ & $0(0)$ & $0(0)$ & \multirow[t]{4}{*}{$N A * *$} \\
\hline $\begin{array}{l}\text { 15-18 (Mild risk) - } \\
\text { No. (\%) }\end{array}$ & $6(4.3)$ & $16(8.8)$ & $11(6.6)$ & & $0(0)$ & $0(0)$ & $0(0)$ & \\
\hline $\begin{array}{l}\text { 13-14 Moderate risk) } \\
\text { - No. (\%) }\end{array}$ & $3(7.7)$ & $4(10.8)$ & $4(9.1)$ & & $0(0)$ & $0(0)$ & $0(0)$ & \\
\hline $\begin{array}{l}\text { 10-12 (High risk) - } \\
\text { No. (\%) }\end{array}$ & $1(16.7)$ & $0(0)$ & $0(0)$ & & $0(0)$ & $0(0)$ & $0(0)$ & \\
\hline \multicolumn{9}{|c|}{ * Percentages may not sum to 100 because of rounding. } \\
\hline \multicolumn{9}{|c|}{$\begin{array}{l}\star \star \\
\text { not posting is not applicable (NA) to Prl incidence rates during the Intervention, because testing incidence of zero events is } \\
\text { not }\end{array}$} \\
\hline \multicolumn{9}{|c|}{$\begin{array}{l}\star \star \star \\
\text { b) }\end{array}$} \\
\hline \multicolumn{9}{|c|}{ † Baseline values are for the 12-month period prior to the start of the Intervention. } \\
\hline \multicolumn{9}{|c|}{$\ddagger$ Intervention values are for the 4-week Intervention period. } \\
\hline \multicolumn{9}{|c|}{$\S$ Indicates repositioning interval for each Intervention Arm. Each Arm included 3 nursing homes. } \\
\hline \multicolumn{9}{|c|}{$\iint$ ANOVA used to test for differences among Arms. } \\
\hline \multicolumn{9}{|c|}{ 9 Chi-square used to test for differences among Arms. } \\
\hline || C-statistic $=0.762$ & & & & & & & & \\
\hline
\end{tabular}

\section{Additional Analyses}

Risk of a Pressure Injury Across and Within Arms

Baseline. Table 3 presents clinical risk (Braden) comparisons, predicted propensity, and observed pressure injury incidence among residents during the Baseline and Intervention. During Baseline, neither first nor mean Braden total risk scores differed by Arm, although risk differed slightly across mean Braden risk categories $(p=0.03)$. Propensity for a resident to develop a pressure injury during Baseline (Table 3) was significantly higher in Arm 2 ( $p<0.001$ ), than either Arm 1 or 3 (Tukey's HSD, P< = 0.05); Arms 1 and 3 did not differ significantly. Odds ratios for pressure injury development during Baseline (Propensity Model C statistic $=0.76)$ included: $\mathrm{Black}$ race $(\mathrm{OR}=2.24 ; \mathrm{Cl}=1.04-4.80)$; Mild Braden risk category $(\mathrm{OR}=3.96 ; \mathrm{Cl}=1.57-9.94)$; and Moderate Braden risk category $(\mathrm{OR}=4.69, \mathrm{Cl}=1.48-14.91)$. No other resident characteristics (age, gender, disease diagnoses) or nursing home or staffing characteristics were significant predictors for developing a pressure injury. Observed annual pressure injury incidence across all nursing homes during Baseline was 5.24\% (52/992; range $=2.3 \%-18.4 \%$ across nursing homes); overall monthly expected number of pressure injuries was 4.33. 
Intervention. No new pressure injuries developed during the Intervention regardless of nursing home allocation to 2, 3, or 4-hour repositioning interval. Despite significant differences in propensity to develop pressure injuries, the incidence rate was $0.0 \%$ across all Arms that included 52 residents with a prior Baseline pressure injury that had healed before the start of the Intervention. Propensity to develop a pressure injury during the Intervention was significantly greater (50\%) at 3 hours (Tukey's HSD, $p \leq 0.05$ ) than at 2 or 4 hours, which were not different.

During the Intervention, neither first nor mean Braden total risk scores differed by Arm. However, both first $(p=0.003)$ and mean $(p=0.001)$ Braden risk categories (low, mild, moderate, high) differed significantly by Arm. Arm 2 included fewer residents at high risk than Arms 1 and 3.

Baseline vs Intervention across and within Arms. Pairwise comparisons of Baseline and Intervention Mean Braden total scores were calculated for each Arm (Table 3). Mean Braden was significantly worse (pressure injury risk was higher) during the Intervention: Arm 1 ( $t=-0.544, p<.001)$; Arm $2(t=-0.224, p=0.015)$; Arm $3(t=-0.643, p<0.001)$. Residents were more likely to score as high risk during the Intervention overall $(n=66,6.7 \%)$ than in Baseline overall $(n=14,1.4 \%)$.

The Baseline pressure injuries showed that the majority of pressure injuries were among residents with mild and moderate Braden risk. Mean Braden risk score categories of Baseline residents with pressure injuries that healed prior to continuing into the Intervention ( $=52$ ) were low (7), mild (33), moderate (11), or high (1) risk.

\section{Compliance with Repositioning Schedule}

Fidelity to the assigned repositioning interval is presented in Table 4. Residents $(n=369)$ wore sensors without interruption for 28 days; $n=623$ residents wore sensors intermittently due to skin irritation, short-term discharge, refusal, permanent discharge, or death. Intervention residents wore patient monitoring system sensors for an average of 16.06 to 17.44 days. However, all Intervention residents were observed for pressure injury development as part of standard care throughout the 28-day Intervention period (Table 2). 
Table 4

Repositioning Characteristics of Residents ( $\mathrm{N}=992)$ During the 4-Week Intervention Period.

\begin{tabular}{|c|c|c|c|c|c|}
\hline \multirow[t]{2}{*}{ Repositioning Characteristics } & \multirow{2}{*}{$\begin{array}{l}\text { Arm } 1 \\
2 \mathrm{hr}+(\mathrm{N}= \\
319)\end{array}$} & \multirow{2}{*}{$\begin{array}{l}\text { Arm } 2 \\
3 \mathrm{hr}+(\mathrm{N}= \\
323)\end{array}$} & \multirow{2}{*}{$\begin{array}{l}\text { Arm } 3 \\
4 \mathrm{hr}+(\mathrm{N}= \\
350)\end{array}$} & \multirow[t]{2}{*}{$\begin{array}{l}P \\
\text { value }\end{array}$} & \multirow[t]{2}{*}{$\begin{array}{l}\text { F- } \\
\text { Statistic }\end{array}$} \\
\hline & & & & & \\
\hline No. of days sensor worn $\ddagger-$ mean (SD) & $\begin{array}{l}16.35 \\
(10.86)\end{array}$ & $\begin{array}{l}17.44 \\
(10.52)\end{array}$ & $\begin{array}{l}16.06 \\
(10.95)\end{array}$ & 0.224 & $1.50 \S$ \\
\hline Mean differences by $\operatorname{Arm}(\mathrm{Cl})^{\star}$ & $\begin{array}{l}1-2 ;-1.096 \\
(-3.098 \\
0.906)\end{array}$ & $\begin{array}{l}2-3 ; 1.381 \\
(-0.578 \\
3.339)\end{array}$ & $\begin{array}{l}1-3 ; 0.285 \\
(-1.675 \\
2.245)\end{array}$ & & \\
\hline \multirow{2}{*}{$\begin{array}{l}\text { Overall resident daily ( } 24 \text {-hour) average no. of } \\
\text { repositioning Turn Alert cues - mean (SD) }\end{array}$} & $4.98(3.41)$ & $3.08(1.72)$ & $1.98(1.12)$ & \multirow[t]{2}{*}{$\dot{0} 001$} & \multirow{2}{*}{$\begin{array}{l}124.07 \\
\S\end{array}$} \\
\hline & $\mathrm{n}=297 \|$ & $\mathrm{n}=292 \|$ & $\mathrm{n}=281^{\|}$ & & \\
\hline Mean differences by Arm (Cl) * & $\begin{array}{l}1-2 ; 1.899 \\
(1.450 \\
2.347)\end{array}$ & $\begin{array}{l}2-3 ; 1.096 \\
(0.641 \\
1.551)\end{array}$ & $\begin{array}{l}1-3 ; 2.995 \\
(2.542 \\
3.448)\end{array}$ & & \\
\hline \multirow{2}{*}{$\begin{array}{l}\text { Overall resident daily average repositioning Turn } \\
\text { Alert overdue hours per } 24 \text { hours - mean (SD) }\end{array}$} & $5.14(3.59)$ & $3.43(2.48)$ & $2.54(1.79)$ & \multirow[t]{2}{*}{$<.001$} & \multirow[t]{2}{*}{$67.66 \S$} \\
\hline & $\mathrm{n}=297^{\|}$ & $\mathrm{n}=292 \|$ & $\mathrm{n}=281 \|$ & & \\
\hline Mean differences by $\operatorname{Arm}(\mathrm{Cl})$ * & $\begin{array}{l}1-2 ; 1.711 \\
(1.181 \\
2.240)\end{array}$ & $\begin{array}{l}2-3 ; 0.889 \\
(0.351 \\
1.426)\end{array}$ & $\begin{array}{l}1-3 ; 2.599 \\
(2.064 \\
3.134)\end{array}$ & & \\
\hline $\begin{array}{l}\text { Overall resident daily (24-hour) on-time repositioning } \\
\text { compliance } \ddagger, \iint-\text { mean (SD) }\end{array}$ & $0.80(0.15)$ & $0.90(0.11)$ & $0.95(0.07)$ & $<0.001$ & $\begin{array}{l}134.50 \\
\S\end{array}$ \\
\hline Mean differences by Arm (Cl) * & $\begin{array}{l}1-2 ;-0.095 \\
(-0.116 \\
-0.074)\end{array}$ & $\begin{array}{l}2-3 ;-0.047 \\
(-0.067 \\
-0.026)\end{array}$ & $\begin{array}{l}1-3 ;-0.141 \\
(-0.162 \\
-0.121)\end{array}$ & & \\
\hline \multicolumn{6}{|l|}{$\begin{array}{l}\text { Overall resident daily (24-hour) on-time repositioning } \\
\text { compliance } \neq, \iint \text { (by Braden risk category } 9 \text { ) - mean } \\
\text { (SD) }\end{array}$} \\
\hline 19-23 (Low risk) & $0.86(0.10)$ & $0.93(0.10)$ & $0.97(0.05)$ & $<.001$ & $54.03 \S$ \\
\hline Mean differences by $\operatorname{Arm}(\mathrm{Cl})$ * & $\begin{array}{l}1-2 ;-0.068 \\
(-0.094 \\
-0.043)\end{array}$ & $\begin{array}{l}2-3 ;-0.038 \\
(-0.064 \\
-0.012)\end{array}$ & $\begin{array}{l}1-3 ;-0.106 \\
(-0.131 \\
-0.082)\end{array}$ & & \\
\hline 15-18 (Mild risk) & $0.79(0.16)$ & $0.89(0.11)$ & $0.95(0.06)$ & $<.001$ & $59.86 \S$ \\
\hline
\end{tabular}

* Mean Difference displayed pairwise: Arm "x"- Arm "y"; Value of actual pairwise mean difference; Confidence Interval (a, b)

† Indicates repositioning interval for each Intervention Arm. Each Arm included 3 nursing homes.

‡ Total $N=988$ due to missing movement data for 4 residents; Arm 2 missing 3 residents $(n=320)$; Arm 3 missing 1 resident $(n=349)$.

$\S$ ANOVA used to test for differences among Arms.

$\iint$ Mean compliance values imputed for 4 missing residents after determining there is no difference in statistical significance between the ANOVA results with and without imputation.

१ Braden risk category determined from baseline Braden Risk Scores for the week prior to the Intervention.

" No. of residents with 1 or more overdue repositioning Turn Alert cues. 


\begin{tabular}{|c|c|c|c|c|c|}
\hline \multirow[t]{2}{*}{ Repositioning Characteristics } & Arm 1 & Arm 2 & Arm 3 & \multirow{2}{*}{$\begin{array}{l}P \\
\text { value }\end{array}$} & \multirow{2}{*}{$\begin{array}{l}\text { F- } \\
\text { Statistic }\end{array}$} \\
\hline & $\begin{array}{l}2 \mathrm{hr}+(\mathrm{N}= \\
319)\end{array}$ & $\begin{array}{l}3 \mathrm{hr}+(\mathrm{N}= \\
323)\end{array}$ & $\begin{array}{l}4 \mathrm{hr}+(\mathrm{N}= \\
350)\end{array}$ & & \\
\hline Mean differences by $\operatorname{Arm}(\mathrm{Cl})$ * & $\begin{array}{l}1-2 ;-0.099 \\
(-0.133 \\
-0.065)\end{array}$ & $\begin{array}{l}2-3 ;-0.059 \\
(-0.090 \\
-0.028)\end{array}$ & $\begin{array}{l}1-3 ;-0.158 \\
(-0.192 \\
-0.124)\end{array}$ & & \\
\hline 13-14 (Moderate risk) & $0.73(0.16)$ & $0.88(0.13)$ & $0.89(0.100$ & $<0.001$ & $18.32 \S$ \\
\hline Mean differences by $\operatorname{Arm}(\mathrm{Cl})$ * & $\begin{array}{l}1-2 ;-0.149 \\
(-0.218 \\
-0.080)\end{array}$ & $\begin{array}{l}2-3 ;-0.009 \\
(-0.082 \\
0.063)\end{array}$ & $\begin{array}{l}1-3 ;-0.159 \\
(-0.230 \\
-0.087)\end{array}$ & & \\
\hline 10-12 (High risk) & $0.72(0.16)$ & $0.89(0.09)$ & $0.93(0.06)$ & $<.001$ & $24.86 \S$ \\
\hline Mean differences by Arm (Cl) * & $\begin{array}{l}1-2 ;-0.169 \\
(-0.261, \\
-0.076)^{\prime}\end{array}$ & $\begin{array}{l}2-3 ;-0.040 \\
(-0.131 \\
0.051)\end{array}$ & $\begin{array}{l}1-3 ;-0.209 \\
(-0.282 \\
-0.136)\end{array}$ & & \\
\hline \multicolumn{6}{|c|}{ * Mean Difference displayed pairwise: Arm "x"- Arm "y"; Value of actual pairwise mean difference; Confidence Interval $(a, b)$} \\
\hline \multicolumn{6}{|c|}{ † Indicates repositioning interval for each Intervention Arm. Each Arm included 3 nursing homes. } \\
\hline \multicolumn{6}{|c|}{$\begin{array}{l}\text { ‡ Total } N=988 \text { due to missing movement data for } 4 \text { residents; Arm } 2 \text { missing } 3 \text { residents }(n=320) \text {; Arm } 3 \text { missing } 1 \\
\text { resident }(n=349) \text {. }\end{array}$} \\
\hline \multicolumn{6}{|c|}{$\S$ ANOVA used to test for differences among Arms. } \\
\hline \multicolumn{6}{|c|}{$\begin{array}{l}\iint_{\text {significance between the ANOVA results with and without imputation. }} \text { Mean compliance values imputed for } 4 \text { missing residents after determining there is no difference in statistical } \\
\text { s.t. }\end{array}$} \\
\hline \multicolumn{6}{|c|}{ Graden risk category determined from baseline Braden Risk Scores for the week prior to the Intervention. } \\
\hline
\end{tabular}

Overall, 24-hour average repositioning cues and average overdue hours were significantly higher for the 2-hour Arm compared to less frequent repositioning intervals $(p<0.001)$. Overdue hours were twice and Turn Alert cues were more than twice as high for 2-hour compared to 4-hour repositioning.

Overall, daily on-time repositioning compliance was significantly better as the assigned hourly repositioning interval lengthened. Nursing homes allocated to the 4-hour interval had significantly greater compliance (95\%) compared to compliance at 3-hour $(90 \%)$ or 2-hour $(80 \%)$ intervals $(p<0.001)$. Daily average on-time repositioning compliance was lower across all Braden risk categories for the 2-hour arm compared to 3- or 4-hour repositioning schedules $(p<0.001)$.

\section{Discussion}

Pressure injuries did not develop during the Intervention in this c-RCT embedded pragmatic cluster randomized trial involving staff cued to reposition nursing home residents at 2-, 3-, or 4-hour intervals. This represents a decrease from Baseline 4.33 pressure injury monthly incidence to zero in the Intervention despite significantly greater risk (worse mean Braden Total scores), greater number of Braden high risk residents than in Baseline, and 52 residents (27\% Black) who had previously healed pressure injuries. The null hypothesis of no increase in pressure injury rates when extending the repositioning interval to 3 or 4 hours cannot be rejected. Despite this, propensities for pressure injury development across Arms, coupled with absence of pressure injuries during the Intervention, suggest the potential to safely extended repositioning requirements from every 2 hours to every 3 or even 4 hours for most residents, thus facilitating uninterrupted sleep, which is critical to overall health. ${ }^{19}$ Two-hour repositioning is the standard of care implemented during the Baseline. Thus, the 2-hour Intervention Arm could not be directly compared to 3- or 4-hour protocols because no change occurred in its repositioning interval between 
Baseline and Intervention. However, study results support relaxation of this 2-hour Arm protocol based on its similar propensity for pressure injury development compared to the 4-hour Arm.

The TEAM-UP study suggests that resident repositioning intervals can be safely extended up to every 4 hours without increasing pressure injury incidence among residents at a wide range of clinical risk, if residents are supported by viable high density foam mattresses and staff are compliant with repositioning. DeFloor and colleagues found that those turned every 4hours on high density foam mattresses experienced significantly fewer pressure injuries than those turned every 2- or 3-hours on standard hospital mattresses. ${ }^{14}$ Prior research studying only moderate and high risk residents using high density foam mattresses found no significant difference in pressure injury incidence with repositioning at 2, 3, or 4 hours. ${ }^{13}$ Residents deemed to be low risk are not commonly studied, yet these residents develop pressure injuries ${ }^{13-15}$, thus, low/mild risk residents were included in TEAM-UP. Fifty-two residents with healed Baseline pressure injuries who participated in the Intervention included $77 \%$ who were of low/mild risk.

The TEAM-UP study buttresses evidence for efficacy of high density foam mattress in preventing pressure injuries for 28 days, even with extended repositioning intervals that were associated with significantly better staff compliance. Also, on-time repositioning was supported by education and cueing staff. Education sessions refreshed staff on etiology of pressure injuries, the importance of tissue offloading, and proper repositioning techniques leading to a heightened awareness of prevention standard protocols already in place. This increased awareness was present across all study Arms, yet compliance was lower in the 2-hour interval because of nursing staff difficulty achieving that frequency.

A variety of cueing reminders have been used to improve staff repositioning compliance; for example, bedside logs, ${ }^{14}$ musical cues, ${ }^{20}$ and paper clocks. ${ }^{13}$ Cueing used in TEAM-UP is thought to be a factor that helped facilitate nursing staff in repositioning on-time. Comparison of repositioning compliance between the monitored every 2-hour Intervention interval and

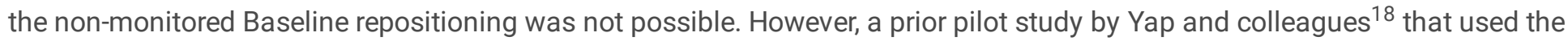
same patient monitoring system reported a mean of $61.4 \%$ repositioning compliance during a 3-day blinded every 2-hour repositioning Baseline without cueing. Repositioning compliance improved to 81.5\% during 18-days of monitoring with cueing, which is similar to TEAM-UP finding of mean $80 \%$ repositioning compliance for the 2-hour Arm. Also, Pickham and colleagues ${ }^{21}$ found a $54 \%$ repositioning compliance in a 2-hour control group of hospitalized acutely ill adults. Similar to TEAM-UP, staff repositioning compliance for hospitalized acutely ill adults was improved by displaying on monitors (visual cues) repositioning information received from wearable sensors. ${ }^{21}$

\section{LIMITATIONS}

TEAM-UP had several limitations. Cluster trials in a healthcare setting, without extensive preliminary analyses, have a large degree of uncertainty related to the within-cluster correlation and between cluster variation. Small differences in an Intraclass Correlation Coefficient can result in substantial differences in estimates of the required sample size and number of clusters. The Intraclass Correlation Coefficient estimate indicated that this study required five nursing homes per Arm; however, the wide confidence interval $(\mathrm{Cl}=-0.78,0.89)$ is evidence of a lack of precision in this measure and does not provide sufficient guidance to determine the optimum number of required nursing homes. The use of a large number of nursing homes is problematic when the goal is to efficiently test multiple repositioning intervals in a real-world setting. The number of nursing homes in the TEAM-UP trial was limited to a total of 9 in order to adhere to the 5-year study period and budget parameters imposed by the funding mechanism while ensuring that the embedded pragmatic cluster randomized trial design requirements were met. This was especially true for implementing the nursing-home-wide Intervention that could be incorporated easily into routine clinical workflow as standard of care.

Propensity score analysis partially addressed differences in resident characteristics between nursing homes in Arms 1,2 , and 3. One source of imbalance in Arms was associated with Blacks being more likely to develop a pressure injury; yet no pressure injuries developed among Black residents during the Intervention. Propensity analysis did not control for variation in a resident's length of stay. Potential bias that might have been introduced by differences in short stay (Length of Stay $<100$

Page $17 / 21$ 
days) and long stay (Length of Stay > 100 days) residents as defined according to Centers for Medicare and Medicaid could not be fully identified based on available data.

This trial excluded nursing home residents with severe pressure injury risk because their care delivery is highly individualized using specialized surfaces and repositioning intervals. Evidence regarding median time to pressure injury development varies; for example, recent acute care evidence shows a 2-5 day median time to pressure injury development when using high density foam mattresses and 4-hour repositioning. ${ }^{22}$ TEAM-UP's 28-day follow-up period was longer than prior nursing home randomized control trials' intervention periods. ${ }^{13,14}$ Prior research supports the adequacy of the 4-week Intervention time period to permit development of pressure injuries in the sample studied. ${ }^{13,14}$ However, this may still be insufficient time to fully demonstrate pressure injury outcomes, precluding using time-to-development as an analysis strategy, as in the PRESSURE2 study. ${ }^{23}$ It was not possible to blind nursing staff to the Intervention. Staff knowledge of the nursing-home-wide repositioning interval was essential but may have contributed to a Hawthorne effect because the patient monitoring system made staff continually aware of resident repositioning needs.

\section{Conclusions}

This study found that a large group of residents could have repositioning protocols relaxed without compromising pressure injury incidence, although this embedded cluster randomized clinical trial could not establish a true causal link between three repositioning interval treatments and pressure injury outcomes. This trial is the third study to demonstrate that pressure injury incidence is not compromised by repositioning most nursing home residents at 3 or 4 hour intervals, ${ }^{13,14,16}$ also, the Intervention period was longer than in previous studies. Residents did not develop new pressure injuries while using viable high density foam mattresses and staff were cued to perform scheduled repositioning, demonstrating, as in prior research, ${ }^{18,24}$ that consistently implemented prevention strategies can be effective. Successful application of these results nursing-homewide would free staff for additional care activities and reduce resident sleep disruptions. Additional research is needed to identify specific factors of race and risk differences in pressure injury incidence. Establishing pressure injury causative factors will enhance quality of nursing home care delivery.

\section{Declarations}

\section{Ethics approval and consent to participate}

The trial was approved by Duke University Institutional Review Board (IRB) (Duke IRB\# Pro00069413). The IRB approved a waiver of informed consent per the United States Department of Health and Human Services guidelines 21 CFR 46 was

granted because: 1) a nursing-home-wide repositioning schedule was provided to the entire cluster of low-, mild-, moderate-, and high-risk residents, 2) the Intervention assigned repositioning interval was adopted as part of nursing-home-wide practice that standardized the repositioning workflow, 3) minimal risk was involved in the cluster-level intervention, and 4) a coded data set was created with assigned study identification (ID) number for resident data extracted from patient monitoring system database, Minimum Data Set, and electronic health record and the coded data set was placed directly into a secured network folder. Also, fliers summarizing the project with notation of nursing home Medical Director approval were mailed to residents and family member/responsible party informing them of the option to choose not to participate and the Principal Investigator's mobile phone number for concerns and questions. The Principal Investigator guided on-site data collection with the Project Director, who was responsible for implementation fidelity. The research team was responsible for data quality control/analyses. A Business Associates Agreement was signed between the nursing home corporation and patient monitoring system company.All methods were performed in accordance with the relevant guidelines and regulations (Declaration of Helsinki).

\section{Consent for publication}

Not Applicable. 
The data used in this publication include protected health information, and therefore cannot be freely shared. Data sharing will be possible with case-by-case approval from our institution's Institutional Review Board; requests may be directed to the corresponding author.

\section{Competing interests}

The authors have no competing interests to declare.

\section{Funding}

Research reported in this publication is supported by the National Institute of Nursing Research of the National Institutes of Health under Award NumberR01NR016001. The content is solely the responsibility of the authors and does not necessarily represent the official views of the National Institutes of Health.

\section{Authors' contributions}

TY, SK, and SH, conceived of the study idea and designed the study. TY, SK, SH, SH, PS, and TZ implemented the analysis plan. All authors contributed to data interpretation and co-wrote the manuscript.

\section{Acknowledgements}

This project was funded by the National Institutes of Health, National Institute

of Nursing Research (R01NR016001) Yap, PI. Authors would like to thank Judith Hayes, PhD, RN and Elizabeth Flint, PhD for editorial assistance.

This project was funded by the National Institutes of Health, National Institute

of Nursing Research (R01NR016001) Yap, Pl. Authors would like to thank Judith Hayes, PhD, RN and Elizabeth Flint, PhD for editorial assistance.

\section{References}

1. National Pressure Ulcer Advisory Panel, European Pressure Ulcer Advisory Panel, Pan Pacific Pressure Injury Alliance, Haesler EE (eds.). Prevention and treatment of pressure ulcers: clinical practice guideline, 2nd ed. Perth, Australia: Cambridge Media; 2014.

2. McInnes E, Jammali-Blasi A, Bell-Syer SE, Dumville JC, Middleton V, Cullum N. Support surfaces for pressure ulcer prevention. Cochrane Database Syst Rev. 2015 Sep 3;2015(9):CD001735.

3. Chaboyer W, Bucknall T, Webster J, et al. The effect of a patient centred care bundle intervention on pressure ulcer incidence (INTACT): a cluster randomised trial. Int J Nurs Stud 2016;64:63-71.

4. Coleman S, Gorecki C, Nelson EA, et al. Patient risk factors for pressure ulcer development: systematic review. Int J Nurs Stud 2013;50:974-1003.

5. Medicare.gov Nursing Home Compare. Retrieved November 9, 2020, from https://www.medicare.gov/nursinghomecompare/search.html?

6. Moore Z. US Medicare data show incidence of hospital-acquired pressure ulcers is $4.5 \%$, and they are associated with longer hospital stay and higher risk of0 death. Evid Based Nurs 2013;16:118-119.

7. Padula WV, Delarmente BA. The national cost of hospital-acquired pressure injuries in the United States. Int Wound J 2019;16:634-640. 
8. Braden BJ, Maklebust J. Preventing pressure ulcers with the Braden scale: an update on this easy-to-use tool that assesses a patient's risk. Am J Nurs 2005;105:70-2.

9. Jalali R, Rezaie M. Predicting pressure ulcer risk: comparing the predictive validity of 4 scales. Adv Skin Wound Care. 2005;18:92-7.

10. Thompson D. An evaluation of the Waterlow pressure ulcer risk-assessment tool. Br J Nurs 2005;14:455-9.

11. Gillespie BM, Walker RM, Latimer SL, et al. Repositioning for pressure injury prevention in adults. Cochrane Database Syst Rev. 2020 Jun 2;6:CD009958.

12. Sharp CA, Schulz Moore JS, McLaws ML. Two-hourly repositioning for prevention of pressure ulcers in the elderly: patient safety or elder abuse? J BioethInq 2019;16:17-34.

13. Bergstrom N, Horn SD, Rapp MP, Stern A, Barrett R, Watkiss M. Turning for Ulcer ReductioN: a multisite randomized clinical trial in nursing homes. J Am Geriatr Soc2013;61:1705-1713.

14. Defloor T, De Bacquer D, Grypdonck MH. The effect of various combinations of turning and pressure reducing devices on the incidence of pressure ulcers. Int J Nurs Stud 2005;42:37-46.

15. Norton D, McLaren R, Exton-Smith AN. An investigation of geriatric nursing problems in hospital: Edinburgh: Churchill Livingston; 1975.

16. Yap TL, Kennerly SM, Horn SD, Bergstrom N, Datta S, Colón-Emeric C. TEAM-UP for quality: a cluster randomized controlled trial protocol focused on preventing pressure ulcers through repositioning frequency and precipitating factors. BMC Geriatr 2018;18:54.

17. Weinfurt, K. (2017). "The Embedded Pragmatic Clinical Trial Ecosystem." What is a Pragmatic Clinical Trial? Retrieved May 18, 2021, from https://rethinkingclinicaltrials.org/chapters/pragmatic-clinical-trial/what-is-a-pragmatic-clinical-trial$2 /$.

18. Yap L, Kennerly SM, Ly K.Pressure injury prevention: outcomes and challenges to use of resident monitoring technology in a nursing home. J Wound Ostomy Continence Nurs 2019;46:207-213.

19. Irwin MR. Sleep and inflammation: partners in sickness and in health. Nat Rev Immunol. 2019;19(11):702-715.

20. Yap TL, Kennerly SM, Simmons MR et al. Multidimensional team-based intervention using musical cues to reduce odds of facility-acquired pressure ulcers in long-term care: a paired randomized intervention study. J Am Geriatr Soc 2013;61:1552-9.

21. Pickham D, Berte N, Pihulic M, Valdez A, Mayer B, Desai M. Effect of a wearable patient sensor on care delivery for preventing pressure injuries in acutely ill adults: a pragmatic randomized clinical trial (LS-HAPI study). Int J Nurs Stud 2018;80:2-19.

22. Jiang Q, Liu Y, Yu H, et al. A Multicenter, Comparative Study of Two Pressure-Redistribution Mattresses with Repositioning Intervals for Critical Care Patients. Adv Skin Wound Care. 2020;33(3):1-9.

23. Brown S, Smith IL, Brown JM. Pressure RElieving Support SUrfaces: a Randomised Evaluation 2 (PRESSURE 2): study protocol for a randomised controlled trial. Trials 2016;17:604.

24. Courvoisier DS, Righi L, Béné N, Rae AC, Chopard P. Variation in pressure ulcer prevalence and prevention in nursing homes: A multicenter study. Appl Nurs Res. 2018 Aug;42:45-50. doi: 10.1016/j.apnr.2018.06.001. Epub 2018 Jun 5. PMID: 30029713.

\section{Figures}




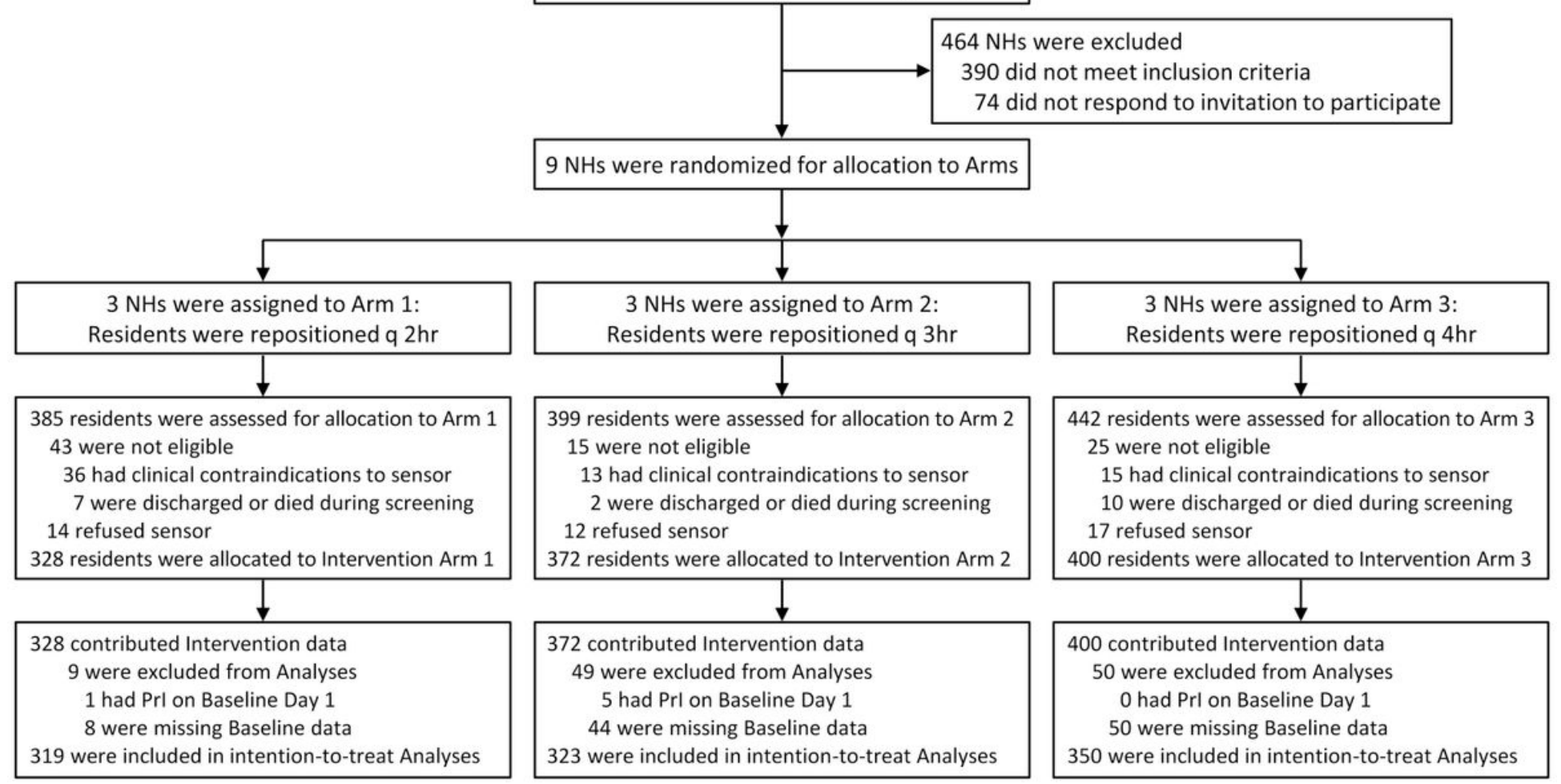

\section{Figure 1}

Nursing Home Enrollment and Randomization, Resident Assessment and Allocation, Follow-up, and Analysis. NHs denotes nursing homes. 